\title{
DOES THE EVALUATION OF COAGULATION FACTORS CONTRIBUTE TO ETIOLOGICAL DIAGNOSIS OF PLEURAL EFFUSIONS?
}

\author{
Marcelo Alexandre Costa Vaz, ${ }^{\mathrm{I}}$ Francisco Suso Vargas, ${ }^{\mathrm{I}}$ Felipe Costa de \\ Andrade Marinho, ' Élbio Antonio D'Amico, "I Tânia Rubia Flores Rocha, ${ }^{\text {II }}$ \\ Lisete Ribeiro Teixeira ${ }^{\mathrm{I}}$
}

doi: $10.1590 / \mathrm{S1807-59322009000900010}$

Costa Vaz MA, Vargas FS, Marinho FCA, D'Amico EA, Rocha TRF, Teixeira LR. Does the evaluation of coagulation factors contribute to etiological diagnosis of pleural effusions?. Clinics. 2009;64(9):891-5.

OBJECTIVE: The aim of this study was to identify the participation of the coagulation system in the differential diagnosis of pleural effusions.

INTRODUCTION: Imbalance between immunologic and metabolic factors triggers a sequence of events resulting in pleural reactions and accumulation of fluid. The coagulation system, which is fundamental for the maintenance of homeostasis, contributes to the inflammatory process responsible for pleural effusions, and participates in cellular proliferation and migration as well as in the synthesis of inflammatory mediators.

METHODS: We evaluated the laboratory profile of coagulation and fibrinolysis in 54 pleural fluids ( 15 transudates and 39 exudates). RESULTS: The coagulation system acts according to the pathophysiologic mechanisms involved in the development of pleural effusions. In inflammatory effusions (exudates), there is activation of coagulation with increased levels of fragment $1+2$ and thrombin-antithrombin complex in addition to reduction of fibrinogen levels due to fibrinolysis and fibrin tissue incorporation. As a consequence, there is activation of the fibrinolytic system with increased levels of fibrin degradation products, including the D-dimer. These changes are not sufficient for differentiation of different subgroups of exudates. In transudates, these events were observed to a lesser degree.

CONCLUSION: The coagulation system plays an important role in the development of pleural diseases. Coagulation tests show differences between transudates and exudates but not among exudate subgroups. Understanding the physiopathological mechanisms of pleural disorders may help to define new diagnostic and therapeutic approaches.

KEYWORDS: Pleural diseases; Coagulation; Fibrinolysis; Transudate; Exudate.

\section{INTRODUCTION}

The pleural layers covering the lungs (visceral) and the thoracic wall (parietal) define the pleural cavity, which is responsible for several important functions, including the maintenance of homeostasis. In this context, the interaction

${ }^{\text {I }}$ Pleural Laboratory, Pulmonary Division, Heart Institute (InCor), Hospital das Clinicas da Faculdade de Medicina da Universidade de São Paulo - São Paulo/SP, Brazil.

II Hemostasis Laboratory, Hematology Service, Hospital das Clinicas da Faculdade de Medicina da Universidade de São Paulo - São Paulo/SP, Brazil. Email: lisetepneumo@yahoo.com.br

Tel: 55113069.5034

Received for publication on March 18, 2009

Accepted for publication on June 30, 2009 between immunologic and metabolic pleural factors should be considered. Their imbalance triggers a sequence of events that generally culminates with the development of a pleural effusion. ${ }^{1}$ According to the pathologic changes, the accumulated fluid is classified as a transudate (an imbalance between the hydrostatic and oncotic pressures in a normal pleura) or an exudate (an inflammatory process with pleural injury).

Characteristically, an exudative effusion demonstrates increased cellularity, protein levels and inflammatory markers. ${ }^{2,3}$ The contribution of the coagulation system is fundamental to the maintenance of homeostasis and should be considered due to its close relationship to the inflammatory process. The coagulation system prevents 
hemorrhage and initiates tissue repair and remodeling, in addition to orchestrating cellular proliferation, cellular migration and synthesis of inflammatory mediators. The coagulation system, when chronically activated and in the presence of an inflammatory state, can generate adverse effects such as chronic release of procoagulant factors (e.g., tissue factor), cellular activation (adhesion molecules), protein modulation (transformation of fibrinogen into fibrin), and even histological changes promoted by cytokines. ${ }^{4}$ All these changes in exudative effusions contribute to tissue remodeling and lead to pleural thickening, trapped lung and functional impairment. ${ }^{4}$ It is worth noting that chronic transudates, which have no inflammatory response, are rarely associated with pleural thickening. ${ }^{1}$ To recognize the contribution of the coagulation system to the differential diagnosis of pleural effusions, we evaluated the laboratory profiles of coagulation and fibrinolysis in pleural fluids of different etiologies.

\section{MATERIALS AND METHODS}

The present study was approved by the Research Ethics Committee of the University and informed written consent was obtained from the patients. Fifty-four patients with a diagnosed pleural effusion were sequentially selected: 15 transudates and 39 exudates (9 parapneumonic, 15 tuberculosis and 15 cancers). Inclusion was based on the presence of a defined etiology and the absence of coagulation disturbances: normal serum values for thrombin time (TT), activated partial thromboplastin time (APTT) and prothrombin time (PT). The presence of an associated pneumothorax determined exclusion.

The patients underwent thoracocentesis and, in some cases, a parietal pleural biopsy. Blood and fluid samples were collected at same time to differentiate transudate from exudates based on Light's criteria. ${ }^{2}$ The etiological diagnosis of the exudates was established by considering, in addition to the clinical parameters, the following criteria:

* Parapneumonic: radiological study compatible with pneumonia, neutrophilic pleural fluid with a positive culture or the presence of bacteria.

* Tuberculosis: lymphocytic fluid ( $>80 \%)$ with rare mesothelial cells $(<5 \%)$ and an elevated adenosine deaminase (> $40 \mathrm{Ul}$ ). The presence of Mycobacterium tuberculosis in the fluid, in the pleural fragment and/or granuloma (with or without caseation) in the histology.

* Malignant: positive oncotic cytology in the fluid or in the pleural fragment.

Samples of blood and pleural fluid were collected in siliconated tubes containing citrate. Thrombin time, activated partial thromboplastin time and prothrombin time were immediately evaluated. Samples of fluid were centrifuged and frozen at $-80^{\circ} \mathrm{C}$ for later measurement of fibrinogen, prothrombin fragment $1+2(\mathrm{~F} 1+2)$, thrombinantithrombin complex (TAT), fibrin degradation products (FDP) and D-dimer levels (enzymatic reaction - ELISA with photometric reading).

\section{Statistical analysis}

Data are presented as means and standard error. To compare the groups, Kruskal-Wallis non-parametric analysis of variance followed by Dunn's test was performed. The power of the study was calculated to 0.8 and p-values lower than 0.05 were considered significant. The statistical program used was Systat Software 2006 (Sigma Stat 3.5; California, USA).

\section{RESULTS}

All patients had normal blood coagulation profiles. Irrespective of etiology, the pleural fluid samples showed higher prothrombin times (PT) and activated partial thromboplastin (aPTT) than those observed in blood.

With respect to plasma, the pleural levels of fibrinogen were reduced (transudates and exudates), while in all other tests, the levels were increased. Significant differences were observed in the pleural levels of fibrinogen between transudates and exudates $(\mathrm{p}=0.004)$ and also among transudates and parapneumonic effusions $(\mathrm{p}=0.011)$ secondary to tuberculosis $(p=0.023)$ and cancer $(p=0.005)$. Comparison among the exudates did not show statistically significant differences $(\mathrm{p}=0.214)$; Figure 1 .

For the levels of fragment $1+2$, we noted results inverse to those observed fibrinogen. The transudates presented a significant reduction with respect to the exudates $(p=0.001)$ and the parapneumonic effusions $(\mathrm{p}=0.0037)$ secondary to tuberculosis $(\mathrm{p}<0.001)$ and cancer $(\mathrm{p}=0.002)$. There were no differences among the exudates $(\mathrm{p}=0.391)$; Figure 1 .

The thrombin-antithrombin complex showed changes similar to $\mathrm{F} 1+2$, with a statistically significant increase in exudates as compared to transudates $(\mathrm{p}<0.001)$. Similarly, there were differences among transudates and parapneumonic effusions $(\mathrm{p}=0.017)$ secondary to tuberculosis $(p<0.001)$ and cancer $(p=0.001)$. No significant differences were observed among the exudates $(\mathrm{p}=0.094)$; Figure 2 .

The values for fibrin degradation products were not different between transudates and exudates $(\mathrm{p}=0.42)$, transudates and parapneumonic effusions $(p=0.068)$, or secondary to tuberculosis $(p=0.798)$ and cancer $(p=0.933)$. Similar findings were observed with respect to D-dimer, 

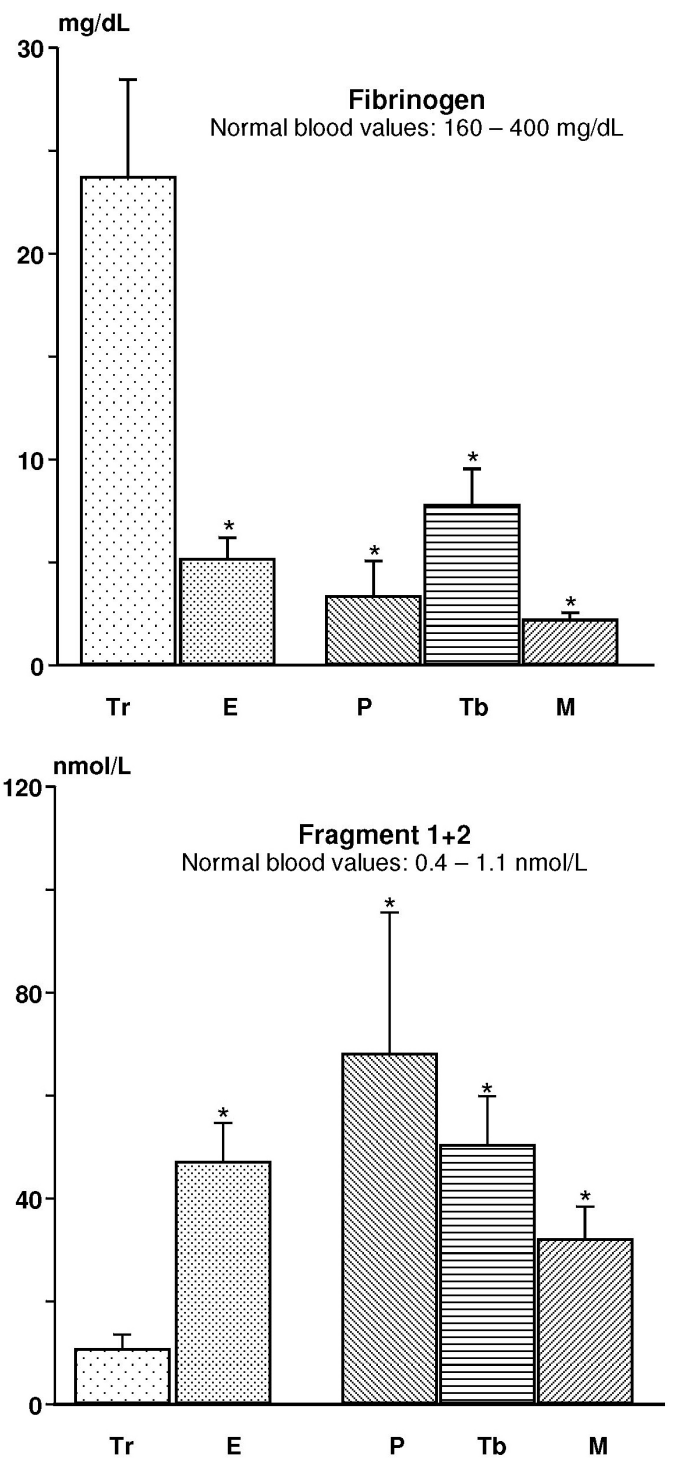

Figure 1 - Fibrinogen and fragment $1+2$ levels (mean \pm standard error) in transudates, exudates $(\mathrm{E})$, parapneumonic $(\mathrm{P})$, tuberculosis $(\mathrm{Tb})$ and cancer (M). $* \mathrm{p}<0.05(\mathrm{x}$ transudate $)$

with no significant differences observed between transudates and exudates $(\mathrm{p}=0.277)$ or parapneumonic effusions $(\mathrm{p}=$ $0.811)$, or secondary to tuberculosis $(\mathrm{p}=0.213)$ and cancer $(\mathrm{p}=0.053)$; Figure 2 .

\section{DISCUSSION}

This study demonstrates that the coagulation system behaves differently in transudates and exudates, reflecting the pathophysiologic mechanisms involved in the development of pleural effusions. This may be an important addition to the methods for its diagnosis. ${ }^{5,6}$

In transudates, there is no increase in protein and cellular permeability, nor is there an inflammatory response or activation of the coagulation cascade. Homeostasis is $\mathrm{ng} / \mathrm{mL}\left(\mathrm{x} 10^{3}\right)$

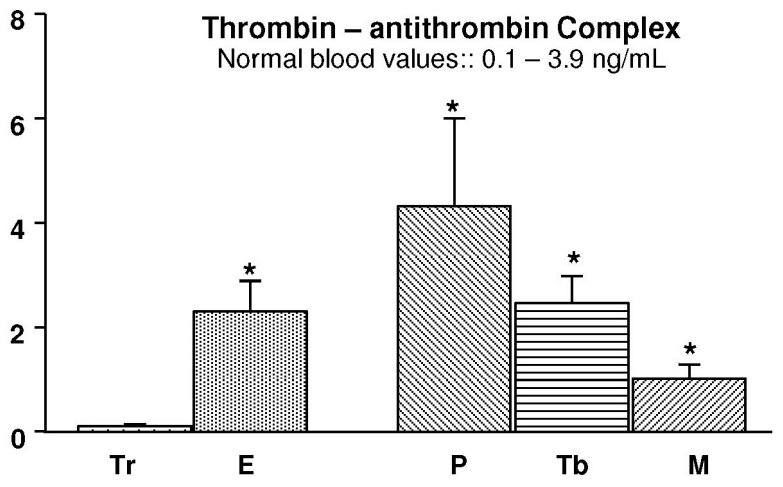

$\mathrm{ng} / \mathrm{mL}\left(\mathrm{x} 10^{3}\right)$

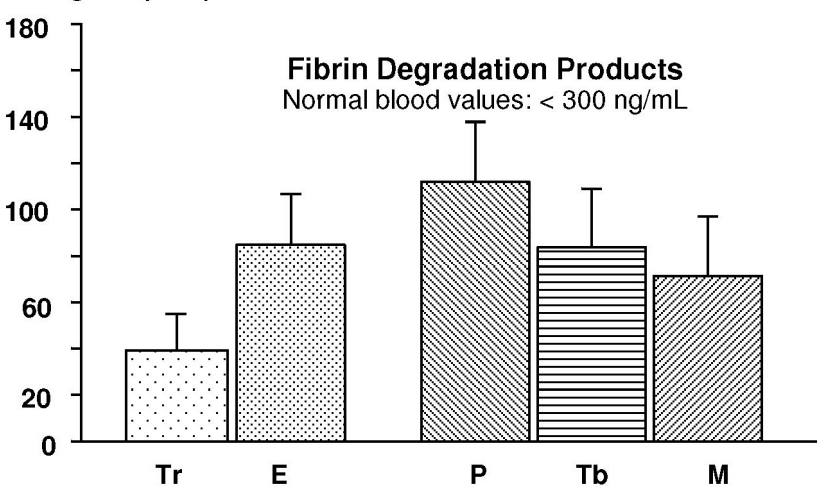

$\mathrm{ng} / \mathrm{mL}\left(\mathbf{x 1 0 ^ { 3 } )}\right.$

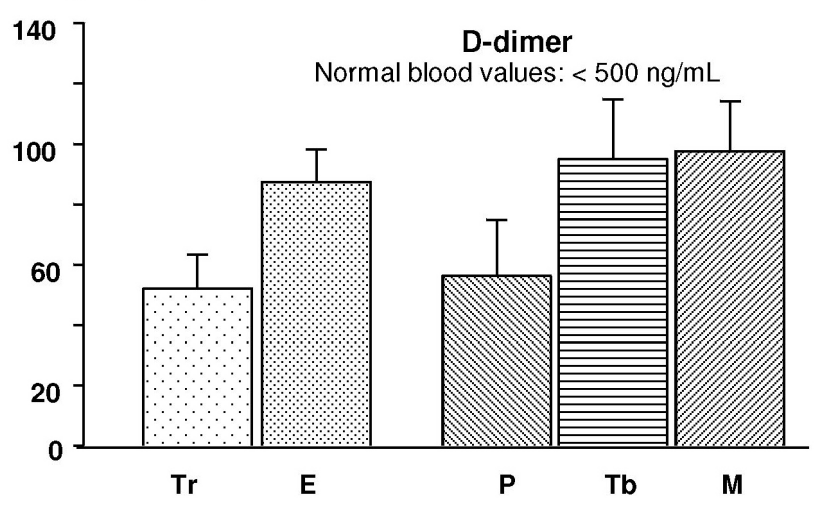

Figure 2 - Thrombin-antithrombin complex (TAT), products of the fibrin degradation (FDP) and D-dimer levels (mean \pm standard error) in transudates, exudates $(\mathrm{E})$, parapneumonic $(\mathrm{P})$, tuberculosis $(\mathrm{Tb})$ and cancer $(\mathrm{M}) .{ }^{*} \mathrm{p}<$ 0.05 (x transudate)

disrupted by the imbalance between pressure and oncotic forces, resulting in fluid accumulation with minimal substrate (proteins) and reduced or absent stimuli for structural changes.

It has been well documented that inflammatory cytokines play important roles in the coagulation and fibrinolytic systems, which, in turn, influence the inflammatory process. This inflammation-coagulation relationship is of fundamental importance to the pathophysiology of pleural 
effusions. The inflammatory response that follows the injury is essential in the tissue repair phase. In this context, fibrin deposition represents the substrate for anatomical reorganization and repair. ${ }^{1,7}$ However, depending on several factors, e.g., the intensity and persistence of injury, simple repair (restitutio ad integrum) may be replaced by fibrosis with pleural thickening and synechiae.

Following tissue injury, vasodilatation secondary to the inflammatory process facilitates the passage of plasma and proteins into the tissue. The binding of tissue factor with factor VII (present in the plasma) forms a complex, activating and initiating the coagulation cascade culminating in the activation of factor $\mathrm{X}$. When this factor acts on prothrombin, it is cleaved into two parts: thrombin and fragment $1+2$; this reflects the activation of coagulation. Thrombin, acting directly on fibrinogen, produces fibrin, which is ultimately responsible for pleural thickening. The maintenance of physiological balance is dependent on coagulation inhibitors (antithrombin, $\mathrm{C}$ and $\mathrm{S}$ proteins). Antithrombin plays an important role by linking itself to thrombin, thus, forming the thrombin-antithrombin complex and blocking thrombin activity. However, once fibrin is formed, the physiological balance is weighted toward lysis (fibrinolysis). In the presence of fibrinolytic activity, an increase in FDP and D-dimer values is observed; the latter being specific to the lysis of polymerized and stabilized fibrin. Inflammatory processes may interfere in the coagulation cascade modifying the reparatory tissue response. ${ }^{8,9}$ In parapneumonic or hemorrhagic effusions, for example, the exaggerated deposition of fibrin frequently produces pleural thickening and septations or loculations. ${ }^{1}$

The results obtained show that in pleural effusions, the coagulation and fibrinolysis profiles reflect the status of the pleural layers. Among inflammatory effusions (exudates), there is coagulation activation due to the production of thrombin (characterized by the increase of F1+2) and reduction in the levels of fibrinogen (resulting from its consumption). As expected, this determines the activation of the fibrinolytic (increased levels of FDP and D-dimer) and anticoagulation (increased TAT) systems as a counterbalance to the procoagulation cascade. The increased formation of fibrin is part of the pathophysiologic mechanism of exudates and is confirmed in the analysis of loculated effusions, which show higher levels of inflammatory markers and lower fibrinolytic activity. ${ }^{10}$
We speculate that fibrin tissue incorporation as well as fibrinolytic activity occurring in the pleural cavity and observed predominantly in exudates may explain the low concentrations of fibrinogen and increased levels of D-dimer, fibrin degradation products and fragment $1+2$.

Routine coagulation tests (PT, TT and APTT) are not adequate to recognize hypercoagulable states. However, they are specific for identifying coagulation deficiency factors in patients with hemorrhagic diseases. The patients in this study showed no changes in routine coagulation tests.

The increased levels of fibrin degradation products in malignant and infectious effusions (when compared to transudates) described by Asted et al. ${ }^{9}$ were not observed in the present study. It is of interest that our results, including those obtained with transudates, show increase levels of fibrin degradation products and D-dimer values, likely reflecting homeostatic degradation of coagulation proteins.

Special characteristics have been described for malignant effusions. High levels of fibrin degradation products and fragment $1+2$ (markedly higher than in serum levels) reveal greater activity of the coagulation system in the pleural space, suggesting participation of this system in invasive cancer. ${ }^{4,11,12}$ These findings, which denote a predominance of procoagulant activity, were not demonstrated in the present study.

\section{CONCLUSION}

Based on the results of this study, we conclude that the coagulation system plays an important role in pleural diseases and that the understanding of pathophysiological mechanisms may open possibilities for new diagnostic and therapeutic approaches. Differences were observed between transudates and exudates, but not among subgroups of exudates. In transudates, the lower coagulation protein influx and the decreased degradation/activation reflect a lesser degree of injury and inflammatory activity. Since there is little or no injury, the coagulation system is not activated and less reparative action and remodeling occur, diminishing the probability of pleural thickening or loculations.

More studies are needed to determine whether different etiologies of exudative pleural diseases affect the coagulation system.

\section{REFERENCES}

1. Mutsaers SE, Prele CM, Brody AR, Idell S. Pathogenesis of pleural fibrosis. Respirology. 2004;9:428-40.
2. Light RW, Macgregor MI, Luchsinger PC, Ball WC Jr. Pleural effusions: the diagnostic separation of transudates and exudates. Ann Intern Med. 1972;77:507-13. 
3. Light RW. Fisiopatologia. In Vargas FS, Teixeira LR, Marchi E, editors. Derrame Pleural, $1^{\text {st }}$ ed - São Paulo: Roca; 2004. p. 33-46.

4. Marinho FCA, Takagaki TY. Hypercoagulability and lung cancer. J Bras Pneumol. 2008;34:312-22.

5. Antonangelo L, Vargas FS, Seiscento M, Bombarda S, Teixeira L, Sales RK. Clinical and laboratory parameters in the differential diagnosis of pleural effusion secondary to tuberculosis or cancer. Clinics. 2007;62:585-90.

6. Aguiar LM, Antonangelo L, Vargas FS, Zerbini MC, Sales MM, Uip DE, Saldiva PH. Malignant and tuberculous pleural effusions: immunophenotypic cellular characterization. Clinics. 2008;63:637-44.

7. Dvorak HF. Tumors: wounds that do not heal. Similarities between tumor stroma generation and wound healing. N Engl J Med. 1986;315:1650-59.

8. Idell S, Girard W, Koenig KB, McLarty J, Fair DS. Abnormalities of pathways of fibrin turnover in the human pleural space. Am Rev Respir Dis. 1991;144:187-94.
9. Astedt B, Adielsson G, Mattsson W. Fibrin/fibrinogen degradation products in pleural exudate. The Lancet. 1976;308:414-6.

10. Chung C-L, Chen C-H, Sheu J-R, Chen Y-C, Chang S-C. Proinflammatory cytokines, transforming growth factor-beta1, and fibrinolytic enzymes in loculated and free-flowing pleural exudates. Chest. 2005;128:690-7.

11. Agrenius V, Chmielewska J, Widstrom O, Blomback M. Pleural fibrinolytic activity is decreased in inflammation as demonstrated in quinacrine pleurodesis treatment of malignant pleural effusion. Am Rev Respir Dis. 1989;140:1381-5.

12. Gieseler F, Luhr I, Kunze T, Mundhenke C, Maass N, Erhart T, et al. Activated coagulation factors in human malignant effusions and their contribution to cancer cell metastasis and therapy. Thromb Haemost. 2007:97:1023-30. 
